\title{
GIN AND LEX OF CERTAIN MONOMIAL IDEALS
}

\author{
SATOSHI MURAI* and TAKAYUKI HIBI
}

\begin{abstract}
Let $A=K\left[x_{1}, \ldots, x_{n}\right]$ denote the polynomial ring in $n$ variables over a field $K$ of characteristic 0 with each $\operatorname{deg} x_{i}=1$. Given arbitrary integers $i$ and $j$ with $2 \leq i \leq n$ and $3 \leq j \leq n$, we will construct a monomial ideal $I \subset A$ such that (i) $\beta_{k}(I)<\beta_{k}(\operatorname{Gin}(I))$ for all $k<i$, (ii) $\beta_{i}(I)=$ $\beta_{i}(\operatorname{Gin}(I))$, (iii) $\beta_{\ell}(\operatorname{Gin}(I))<\beta_{\ell}(\operatorname{Lex}(I))$ for all $\ell<j$ and (iv) $\beta_{j}(\operatorname{Gin}(I))=\beta_{j}(\operatorname{Lex}(I))$, where $\operatorname{Gin}(I)$ is the generic initial ideal of $I$ with respect to the reverse lexicographic order induced by $x_{1}>\cdots>x_{n}$ and where $\operatorname{Lex}(I)$ is the lexsegment ideal with the same Hilbert function as $I$.
\end{abstract}

\section{Introduction}

Let $A=K\left[x_{1}, \ldots, x_{n}\right]$ denote the polynomial ring in $n$ variables over a field $K$ of characteristic 0 with each $\operatorname{deg} x_{i}=1$. Let $<_{\operatorname{lex}}$ (resp. $<_{\text {rev }}$ ) denote the lexicographic (resp. reverse lexicographic) order on $A$ induced by the ordering $x_{1}>\cdots>x_{n}$ of the variables. Given a homogeneous ideal $I$ of $A$, we write $\operatorname{Gin}(I)$ for the generic initial ideal ([6, p. 348]) of $I$ with respect to $<_{\text {rev }}$ and $\operatorname{Lex}(I)$ for the lexsegment ideal ([2] and [11]) with the same Hilbert function as $I$. Let $\beta_{i}(I)=\operatorname{dim}_{K} \operatorname{Tor}_{i}^{A}(K, I)$ denote the $i$ th Betti number of $I$ over $A$. It is known that

$$
\beta_{i}(I) \leq \beta_{i}(\operatorname{Gin}(I)) \leq \beta_{i}(\operatorname{Lex}(I))
$$

for all $i \geq 0$. One has $\beta_{i}(I)=\beta_{i}(\operatorname{Gin}(I))$ for all $i$ if and only if $I$ is componentwise linear ([1]). One has $\beta_{i}(I)=\beta_{i}(\operatorname{Lex}(I))$ for all $i$ if and only if $I$ is Gotzmann ([10]). On the other hand, the following facts are due to [5, Corollary 2.7]:

(i) If $\beta_{i}(I)=\beta_{i}(\operatorname{Gin}(I))$ for some $i$, then $\beta_{k}(I)=\beta_{k}(\operatorname{Gin}(I))$ for all $k \geq i$;

(ii) If $\beta_{i}(I)=\beta_{i}(\operatorname{Lex}(I))$ for some $i$, then $\beta_{k}(I)=\beta_{k}(\operatorname{Lex}(I))$ for all $k \geq i$.

These behaviors of Betti numbers of $\operatorname{Gin}(I)$ and $\operatorname{Lex}(I)$ would naturally lead us to present the following

Question 0.1. Given arbitrary integers $1 \leq i \leq j \leq n$, does there exist a monomial ideal $I$ of $A=K\left[x_{1}, \ldots, x_{n}\right]$ with the properties that

\footnotetext{
* The author is supported by JSPS Research Fellowships for Young Scientists.
}

Received October 10, 2005. 
(i) $\beta_{k}(I)<\beta_{k}(\operatorname{Gin}(I))$ for all $k<i$;

(ii) $\beta_{i}(I)=\beta_{i}(\operatorname{Gin}(I))$;

(iii) $\beta_{\ell}(I)<\beta_{\ell}(\operatorname{Lex}(I))$ for all $\ell<j$;

(iv) $\beta_{j}(I)=\beta_{j}(\operatorname{Lex}(I))$ ?

The above Question 0.1 does ask the relation between the Betti numbers of $I$ and those of $\operatorname{Gin}(I)$ together with the relation between the Betti numbers of $I$ and those of $\operatorname{Lex}(I)$. In the present paper, however, we study the relation between the Betti numbers of $I$ and those of $\operatorname{Gin}(I)$ together with the relation between the Betti numbers of $\operatorname{Gin}(I)$ and those of $\operatorname{Lex}(I)$. Our goal is to show the following

Theorem 0.2. Let $A=K\left[x_{1}, \ldots, x_{n}\right]$ denote the polynomial ring in $n$ variables over a field $K$ of characteristic 0 with each $\operatorname{deg} x_{i}=1$. Given arbitrary integers $i$ and $j$ with $2 \leq i \leq n$ and $3 \leq j \leq n$, there exists $a$ monomial ideal $I \subset$ A such that

(i) $\beta_{k}(I)<\beta_{k}(\operatorname{Gin}(I))$ for all $k<i$;

(ii) $\beta_{i}(I)=\beta_{i}(\operatorname{Gin}(I))$;

(iii) $\beta_{\ell}(\operatorname{Gin}(I))<\beta_{\ell}(\operatorname{Lex}(I))$ for all $\ell<j$;

(iv) $\beta_{j}(\operatorname{Gin}(I))=\beta_{j}(\operatorname{Lex}(I))$.

A monomial ideal required in Theorem 0.2 will be given in Section 2 in case of $2 \leq i<j \leq n$ and in Section 3 in case of $3 \leq j \leq i \leq n$. On the other hand, in Section 1, the reason why the assumption $3 \leq j$ is indispensable in Theorem 0.2 will be explained.

\section{Betti numbers of strongly stable ideals}

Let $A=K\left[x_{1}, \ldots, x_{n}\right]$ denote the polynomial ring in $n$ variables over a field $K$ of characteristic 0 with each $\operatorname{deg} x_{i}=1$. Recall that a monomial ideal $I$ of $A$ is strongly stable if for any monomial $u x_{p} \in I$ and $q<p$ we have $u x_{q} \in I$. Since the base field $K$ is of characteristic 0 , the generic initial ideal $\operatorname{Gin}(I)$ of an arbitrary homogeneous ideal of $A$ is strongly stable ([6, Theorem 15.23]).

Lemma 1.1 below explains the reason why the assumption $3 \leq j$ is indispensable in Theorem 0.2.

Lemma 1.1. Let $I \subset A$ be a strongly stable monomial ideal and suppose that $\beta_{i}(I)=\beta_{i}(\operatorname{Lex}(I))$ for all $i \geq 2$. Then $\beta_{i}(I)=\beta_{i}(\operatorname{Lex}(I))$ for all $i \geq 0$.

Proof. Let $I$ be a strongly stable ideal. We write $m_{\leq k}(I, j)$ for the number of those monomials $u \in I$ of degree $j$ with $m(u) \leq k$, where $m(u)$ is the largest integer $q$ for which $x_{q}$ divides $u$. The computation of the Betti numbers 
of a strongly stable ideal can be done by using the formula [2, Proposition 2.3] which says that

$$
\beta_{i, i+j}(I)=C-D,
$$

where

$$
\begin{aligned}
C & =m_{\leq n}(I, j)\left(\begin{array}{c}
n-1 \\
i
\end{array}\right), \\
D & =\sum_{k=i}^{n-1} m_{\leq k}(I, j)\left(\begin{array}{c}
k-1 \\
i-1
\end{array}\right)+\sum_{k=i+1}^{n} m_{\leq k}(I, j-1)\left(\begin{array}{c}
k-1 \\
i
\end{array}\right) .
\end{aligned}
$$

Since $\operatorname{Lex}(I)$ and $I$ have the same Hilbert function, one has $m_{\leq n}(\operatorname{Lex}(I), j)=$ $m_{\leq n}(I, j)$ for all $j$. In addition, it is known [2, Theorem 2.1] that $m_{\leq k}(\operatorname{Lex}(I), j) \leq m_{\leq k}(I, j)$ for all $j$ and $k$. Thus, since $\beta_{i, i+j}(I)=$ $\beta_{i, i+j}(\operatorname{Lex}(I))$ for all $i \geq 2$ and for all $j$, it follows that $m_{\leq k}(\operatorname{Lex}(I), j)=$ $m_{\leq k}(I, j)$ for all $j$ and for all $k \geq 2$. On the other hand, since $I$ is strongly stable, one has $m_{\leq 1}(I, j)=1$ unless $I_{j}=(0)$, where $I_{j}$ is the $j$ th graded component of $I$. Hence $m_{\leq 1}(\operatorname{Lex}(I), j)=m_{\leq 1}(I, j)$ for all $j$. Thus $m_{\leq k}(\operatorname{Lex}(I), j) \leq m_{\leq k}(I, j)$ for all $j$ and for all $k$.

On the other hand, the reason why the assumption $2 \leq i$ is indispensable in Theorem 0.2 is clear. In fact, if $\beta_{1}(I)=\beta_{1}(\operatorname{Gin}(I))$, then $\beta_{i}(I)=\beta_{i}(\operatorname{Gin}(I))$ for all $i \geq 0$. To see why this is true, suppose that $\beta_{1}(I)=\beta_{1}(\operatorname{Gin}(I))$. Then $\beta_{i}(I)=\beta_{i}(\operatorname{Gin}(I))$ for all $i \geq 1$. Since $\beta_{i, i+j}(I) \leq \beta_{i, i+j}(\operatorname{Gin}(I))$ for all $i$ and for all $j$, one has $\beta_{i, i+j}(I)=\beta_{i, i+j}(\operatorname{Gin}(I))$ for all $i \geq 1$ and for all $j$. Since $I$ and $\operatorname{Gin}(I)$ have the same Hilbert function, it follows that $\beta_{0, j}(I)=\beta_{0, j}(\operatorname{Gin}(I))$ for all $j$. Thus in particular $\beta_{0}(I)=\beta_{0}(\operatorname{Gin}(I))$, as desired.

\section{Construction in the case of $2 \leq i<j \leq n$}

Now we split Theorem 0.2 into Theorem 2.1 and Theorem 3.1. A desired monomial ideal in the case of $2 \leq i<j \leq n$ will be given in Theorem 2.1 and that in the case of $3 \leq j \leq i \leq n$ will be given in Theorem 3.1.

THeORem 2.1. Let $A=K\left[x_{1}, \ldots, x_{n}\right]$ denote the polynomial ring in $n$ variables over a field $K$ of characteristic 0 with each $\operatorname{deg} x_{i}=1$. Fix arbitrary integers $i$ and $j$ with $1<i<j \leq n$ and $J$ the monomial ideal of $A$ which is generated by those quadratic monomials $x_{p} x_{q}, 1 \leq p \leq q \leq n$, with $x_{i-1} x_{j}<_{\operatorname{lex}} x_{p} x_{q}$. Let I be the monomial ideal

$$
I=\left(x_{1}, \ldots, x_{n}\right)^{3}+J+\left(x_{n}^{2}\right)
$$


of A. Then one has

(i) $\beta_{k}(I)<\beta_{k}(\operatorname{Gin}(I))$ for all $k<i$;

(ii) $\beta_{i}(I)=\beta_{i}(\operatorname{Gin}(I))$;

(iii) $\beta_{\ell}(\operatorname{Gin}(I))<\beta_{\ell}(\operatorname{Lex}(I))$ for all $\ell<j$;

(iv) $\beta_{j}(\operatorname{Gin}(I))=\beta_{j}(\operatorname{Lex}(I))$.

Proof. First Step. Given a monomial ideal $L$ of $A=K\left[x_{1}, \ldots, x_{n}\right]$ generated by quadratic monomials, we introduce a finite graph $\Gamma(L)$ on the vertex set

$$
V=\left\{1, \ldots, n, 1^{\prime}, \ldots, n^{\prime}\right\}
$$

whose edge set $E(\Gamma(L))$ consists of those edges

(i) $\{p, q\}$ with $1 \leq p<q \leq n$ such that $x_{p} x_{q} \notin L$;

(ii) $\left\{p, q^{\prime}\right\}$ with $1 \leq p \leq n, 1 \leq q \leq n$ and $p \neq q$;

(iii) $\left\{p, p^{\prime}\right\}$ with $1 \leq p \leq n$ such that $x_{p}^{2} \notin L$.

If $W \subset V$, then we write $\Gamma(L)_{W}$ for the induced subgraph of $\Gamma(L)$ on $W$. Let $\delta\left(\Gamma(L)_{W}\right)$ denote the number of connected component of $\Gamma(L)_{W}$.

By using Hochster's formula [3, Theorem 5.5.1] together with the polarization technique [3, Lemma 4.2.16], it follows that the Betti number $\beta_{k, k+2}(L)=$ $\operatorname{dim}_{K}\left[\operatorname{Tor}_{k}^{A}(K, I)\right]_{k+2}$ can be computed by the formula

$$
\beta_{k, k+2}(L)=\sum_{W \subset V,|W|=k+2}\left(\delta\left(\Gamma(L)_{W}\right)-1\right) .
$$

Second Step. Let $\operatorname{Gin}_{<\operatorname{lex}}(I)$ denote the generic initial ideal of $I$ with respect to the lexicographic order $<_{\text {lex }}$. We claim

$$
\begin{aligned}
\operatorname{Gin}(I) & =\left(x_{1}, \ldots, x_{n}\right)^{3}+J+\left(x_{i}^{2}\right) \\
\operatorname{Lex}(I) & =\operatorname{Gin}_{<_{\operatorname{lex}}}(I)=\left(x_{1}, \ldots, x_{n}\right)^{3}+J+\left(x_{i-1} x_{j}\right) .
\end{aligned}
$$

Let the general linear group $\mathrm{GL}(n ; K)$ act linearly on $A$. Let $\psi \in \mathrm{GL}(n ; K)$. Then $\operatorname{Gin}(\psi(I))=\operatorname{Gin}(I)$ and $\operatorname{Gin}_{<_{\operatorname{lex}}}(\psi(I))=\operatorname{Gin}_{<_{\operatorname{lex}}}(I)$.

- Let $\varphi \in \mathrm{GL}(n ; K)$ be defined by $\varphi\left(x_{t}\right)=x_{t}$ for all $t \neq n$ and $\varphi\left(x_{n}\right)=x_{i}+x_{n}$. Then the initial ideal $\operatorname{in}_{<_{\text {rev }}}(\varphi(I))$ of $\varphi(I)$ with respect to $<_{\text {rev }}$ coincides with the strongly stable ideal $\left(x_{1}, \ldots, x_{n}\right)^{3}+J+\left(x_{i}^{2}\right)$. Hence $\operatorname{Gin}\left(\operatorname{in}_{<_{\text {rev }}}(\varphi(I))\right)=\left(x_{1}, \ldots, x_{n}\right)^{3}+J+\left(x_{i}^{2}\right)$. Since in $<_{\text {rev }}(\varphi(I))$ contains all monomials $x_{p} x_{q}, 1 \leq p \leq q \leq n$, with $x_{i}^{2} \leq_{\text {rev }} x_{p} x_{q}$, it follows from [4, Corollary 1.6] that $x_{i}^{2} \in \operatorname{Gin}(\varphi(I))$. Moreover, since $J$ is strongly stable and $J \subset \varphi(I)$, one has $J=\operatorname{Gin}(J) \subset \operatorname{Gin}(\varphi(I))$. Thus $\left(x_{1}, \ldots, x_{n}\right)^{3}+J+\left(x_{i}^{2}\right) \subset$ $\operatorname{Gin}(\varphi(I))=\operatorname{Gin}(I)$. Hence $\operatorname{Gin}(I)=\left(x_{1}, \ldots, x_{n}\right)^{3}+J+\left(x_{i}^{2}\right)$, as desired. 
- Let $\varphi \in \operatorname{GL}(n ; K)$ be defined by $\varphi\left(x_{t}\right)=x_{t}$ for all $t \neq n$ and $\varphi\left(x_{n}\right)=$ $x_{i-1}+x_{j}+x_{n}$. Then the initial ideal $\operatorname{in}_{<_{\operatorname{lex}}}(\varphi(I))$ of $\varphi(I)$ with respect to $<_{\text {lex }}$ coincides with the strongly stable ideal $\left(x_{1}, \ldots, x_{n}\right)^{3}+J+\left(x_{i-1} x_{j}\right)$. Hence $\operatorname{Gin}_{<_{\text {lex }}}\left(\operatorname{in}_{<_{\text {lex }}}(\varphi(I))\right)=\left(x_{1}, \ldots, x_{n}\right)^{3}+J+\left(x_{i-1} x_{j}\right)$. Since in $<_{\text {lex }}(\varphi(I))$ contains all monomials $x_{p} x_{q}, 1 \leq p \leq q \leq n$, with $x_{i-1} x_{j} \leq_{\text {lex }} x_{p} x_{q}$, it follows from [4, Corollary 1.6] that $x_{i-1} x_{j} \in \operatorname{Gin}_{<_{\text {lex }}}(\varphi(I))$. Moreover, since $J$ is strongly stable and $J \subset \varphi(I)$, one has $J=\operatorname{Gin}_{<_{\operatorname{lex}}}(J) \subset \operatorname{Gin}_{<_{\operatorname{lex}}}(\varphi(I))$. Thus $\left(x_{1}, \ldots, x_{n}\right)^{3}+J+\left(x_{i-1} x_{j}\right) \subset \operatorname{Gin}_{<\operatorname{lex}}(\varphi(I))=\operatorname{Gin}_{<\operatorname{lex}}(I)$. Hence $\operatorname{Gin}_{<_{\text {lex }}}(I)=\left(x_{1}, \ldots, x_{n}\right)^{3}+J+\left(x_{i-1} x_{j}\right)$. Since the ideal $J+\left(x_{i-1} x_{j}\right)$ is lexsegment, one has $\operatorname{Lex}(I)=\left(x_{1}, \ldots, x_{n}\right)^{3}+J+\left(x_{i-1} x_{j}\right)$. Hence Lex $(I)=$ $\operatorname{Gin}_{<_{\text {lex }}}(I)=\left(x_{1}, \ldots, x_{n}\right)^{3}+J+\left(x_{i-1} x_{j}\right)$, as desired.

Third Step. We compute

$$
\begin{aligned}
\beta_{k, k+2}(I) & =\beta_{k, k+2}\left(J+\left(x_{n}^{2}\right)\right) \\
\beta_{k, k+2}(\operatorname{Gin}(I)) & =\beta_{k, k+2}\left(J+\left(x_{i}^{2}\right)\right) \\
\beta_{k, k+2}(\operatorname{Lex}(I)) & =\beta_{k, k+2}\left(J+\left(x_{i-1} x_{j}\right)\right),
\end{aligned}
$$

based on the formula (1) together with the combinatorics on the finite graphs $\Gamma(J), \Gamma\left(J+\left(x_{n}^{2}\right)\right), \Gamma\left(J+\left(x_{i}^{2}\right)\right)$ and $\Gamma\left(J+\left(x_{i-1} x_{j}\right)\right)$ with

$$
\begin{gathered}
E\left(\Gamma\left(J+\left(x_{n}^{2}\right)\right)\right)=E(\Gamma(J)) \backslash\left\{\left\{n, n^{\prime}\right\}\right\} ; \\
E\left(\Gamma\left(J+\left(x_{i}^{2}\right)\right)\right)=E(\Gamma(J)) \backslash\left\{\left\{i, i^{\prime}\right\}\right\} ; \\
E\left(\Gamma\left(J+\left(x_{i-1} x_{j}\right)\right)\right)=E(\Gamma(J)) \backslash\{\{i-1, j\}\} .
\end{gathered}
$$

- Let $W \subset V$ with $|W|=k+2$ and suppose that $\delta\left(\Gamma\left(J+\left(x_{n}^{2}\right)\right)_{W}\right)>$ $\delta\left(\Gamma(J)_{W}\right)$. Then (i) both $n$ and $n^{\prime}$ belong to $W$, (ii) no connected component of $\Gamma\left(J+\left(x_{n}^{2}\right)\right)_{W}$ contains both $n$ and $n^{\prime}$, and (iii) $\delta\left(\Gamma\left(J+\left(x_{n}^{2}\right)\right)_{W}\right)=\delta\left(\Gamma(J)_{W}\right)+$ 1. Since $\left\{\alpha, n^{\prime}\right\}$ is an edge of $\Gamma(J)$ for all $\alpha \in V$ with $\alpha \neq n^{\prime}$ and since $\{\beta, n\}$ is an edge of $\Gamma(J)$ if and only if $\beta \in V \backslash\{1,2, \ldots, i-2\}$, it follows that $W \backslash\left\{n, n^{\prime}\right\} \subset\{1,2, \ldots, i-2\}$. Hence the number of subsets $W \subset V$ with $|W|=k+2$ such that $\delta\left(\Gamma\left(J+\left(x_{n}^{2}\right)\right)_{W}\right)>\delta\left(\Gamma(J)_{W}\right)$ is $\left(\begin{array}{c}i-2 \\ k\end{array}\right)$.

- Let $W \subset V$ with $|W|=k+2$ and suppose that $\delta\left(\Gamma\left(J+\left(x_{i}^{2}\right)\right)_{W}\right)>$ $\delta\left(\Gamma(J)_{W}\right)$. Then (i) both $i$ and $i^{\prime}$ belong to $W$, (ii) no connected component of $\Gamma\left(J+\left(x_{n}^{2}\right)\right)_{W}$ contains both $i$ and $i^{\prime}$, and (iii) $\delta\left(\Gamma\left(J+\left(x_{i}^{2}\right)\right)_{W}\right)=\delta\left(\Gamma(J)_{W}\right)+1$. Since $\left\{\alpha, i^{\prime}\right\}$ is an edge of $\Gamma(J)$ for all $\alpha \in V$ with $\alpha \neq i^{\prime}$ and since $\{\beta, i\}$ is an edge of $\Gamma(J)$ if and only if $\beta \in V \backslash\{1,2, \ldots, i-1\}$, it follows that $W \backslash\left\{i, i^{\prime}\right\} \subset\{1,2, \ldots, i-1\}$. Hence the number of subsets $W \subset V$ with $|W|=k+2$ such that $\delta\left(\Gamma\left(J+\left(x_{i}^{2}\right)\right)_{W}\right)>\delta\left(\Gamma(J)_{W}\right)$ is $\left(\begin{array}{c}i-1 \\ k\end{array}\right)$.

- Let $W \subset V$ with $|W|=k+2$ and suppose that $\delta\left(\Gamma\left(J+\left(x_{i-1} x_{j}\right)\right)_{W}\right)>$ $\delta\left(\Gamma(J)_{W}\right)$. Then (i) both $i-1$ and $j$ belong to $W$, (ii) no connected component 
of $\Gamma\left(J+\left(x_{i-1} x_{j}\right)\right)_{W}$ contains both $i-1$ and $j$, and (iii) $\delta\left(\Gamma\left(J+\left(x_{i-1} x_{j}\right)\right)_{W}\right)=$ $\delta\left(\Gamma(J)_{W}\right)+1$. Since $\{\alpha, i-1\}$ is an edge of $\Gamma(J)$ if and only if

$$
\alpha \in\{j, j+1, \ldots, n\} \bigcup\left(\left\{1^{\prime}, 2^{\prime}, \ldots, n^{\prime}\right\} \backslash\left\{(i-1)^{\prime}\right\}\right)
$$

and since $\{\beta, j\}$ is an edge of $\Gamma(J)$ if and only if

$$
\beta \in(\{i-1, i, \ldots, n\} \backslash\{j\}) \bigcup\left\{1^{\prime}, 2^{\prime}, \ldots, n^{\prime}\right\},
$$

it follows that

$$
W \backslash\{i-1, j\} \subset\left\{1,2, \ldots, i-2,(i-1)^{\prime}, i, i+1, \ldots, j-1\right\} .
$$

Hence the number of subsets $W \subset V$ with $|W|=k+2$ such that $\delta(\Gamma(J+$ $\left.\left.\left(x_{i-1} x_{j}\right)\right)_{W}\right)>\delta\left(\Gamma(J)_{W}\right)$ is $\left(\begin{array}{c}j-1 \\ k\end{array}\right)$.

Hence

$$
\begin{aligned}
\beta_{k, k+2}(I) & =\beta_{k, k+2}(J)+\left(\begin{array}{c}
i-2 \\
k
\end{array}\right) ; \\
\beta_{k, k+2}(\operatorname{Gin}(I)) & =\beta_{k, k+2}(J)+\left(\begin{array}{c}
i-1 \\
k
\end{array}\right) ; \\
\beta_{k, k+2}(\operatorname{Lex}(I)) & =\beta_{k, k+2}(J)+\left(\begin{array}{c}
j-1 \\
k
\end{array}\right) .
\end{aligned}
$$

Thus in particular

(i) $\beta_{k, k+2}(I)<\beta_{k, k+2}(\operatorname{Gin}(I))$ for all $k<i$;

(ii) $\beta_{k, k+2}(I)=\beta_{k, k+2}(\operatorname{Gin}(I))$ for all $k \geq i$;

(iii) $\beta_{\ell, \ell+2}(\operatorname{Gin}(I))<\beta_{\ell, \ell+2}(\operatorname{Lex}(I))$ for all $\ell<j$;

(iv) $\beta_{\ell, \ell+2}(\operatorname{Gin}(I))=\beta_{\ell, \ell+2}(\operatorname{Lex}(I))$ for all $\ell \geq j$.

Fourth Step. Since the regularity of each of $I, \operatorname{Gin}(I)$ and $\operatorname{Lex}(I)$ is 3 , it follows that

$$
\beta_{p, p+q}(I)=\beta_{p, p+q}(\operatorname{Gin}(I))=\beta_{p, p+q}(\operatorname{Lex}(I))=0
$$

for all $q>3$. The cancellation principle [8, Corollary 1.21] now guarantees that

$$
\beta_{k, k+2}(\operatorname{Gin}(I))-\beta_{k, k+2}(I)=\beta_{(k-1),(k-1)+3}(\operatorname{Gin}(I))-\beta_{(k-1),(k-1)+3}(I)
$$

for all $k$. Since $\beta_{k, k+2}(I)=\beta_{k, k+2}(\operatorname{Gin}(I))$ for all $k \geq i$, one has

$$
\beta_{(k-1),(k-1)+3}(I)=\beta_{(k-1),(k-1)+3}(\operatorname{Gin}(I))
$$


for all $k-1 \geq i-1$. Hence $\beta_{k}(I)=\beta_{k}(\operatorname{Gin}(I))$ for all $k \geq i$. Similarly, since $\operatorname{Lex}(I)=\operatorname{Gin}_{<_{\operatorname{lex}}}(I)$, by using the cancellation principle to $\operatorname{Gin}_{<_{\operatorname{lex}}}(I)$, one has $\beta_{\ell}(I)=\beta_{\ell}(\operatorname{Lex}(I))$ for all $\ell \geq j$, as required.

\section{Construction in the case of $3 \leq j \leq i \leq n$}

A monomial ideal in the case of $3 \leq j \leq i \leq n$ in Theorem 0.2 will be given in Theorem 3.1. Note that in Theorem 3.1 we use $i+1$ and $j+1$ instead of $i$ and $j$, so that we work with fixing arbitrary integers $i$ and $j$ with $2 \leq j \leq i<n$.

Theorem 3.1. Let $A=K\left[x_{1}, \ldots, x_{n}\right]$ denote the polynomial ring in $n$ variables over a field $K$ of characteristic 0 with each $\operatorname{deg} x_{i}=1$. Fix arbitrary integers $i$ and $j$ with $2 \leq j \leq i<n$. Let $H$ denote the monomial ideal of $A$ which is generated by those quadratic monomials $x_{p} x_{q}, 1 \leq p \leq q \leq n$, with $x_{j-1} x_{j} \leq_{\operatorname{lex}} x_{p} x_{q}$. Let $G$ denote the monomial ideal of $A$ which is generated by those quadratic monomials $x_{p} x_{q}, 2 \leq p \leq q \leq n$, with $x_{i}^{2} \leq_{\operatorname{lex}} x_{p} x_{q}$. Suppose that I is the monomial ideal

$$
I=x_{1}\left(H+\left(x_{n}^{2}\right)\right)+x_{1}\left(x_{1}, \ldots, x_{n}\right)^{3}+x_{2}^{2}\left(G+\left(x_{n}^{2}\right)\right)+\left(x_{1}, \ldots, x_{n}\right)^{5}
$$

of $A$. Then one has

(i) $\beta_{k}(I)<\beta_{k}(\operatorname{Gin}(I))$ for all $k \leq i$;

(ii) $\beta_{i+1}(I)=\beta_{i+1}(\operatorname{Gin}(I))$;

(iii) $\beta_{\ell}(\operatorname{Gin}(I))<\beta_{\ell}(\operatorname{Lex}(I))$ for all $\ell \leq j$;

(iv) $\beta_{j+1}(\operatorname{Gin}(I))=\beta_{j+1}(\operatorname{Lex}(I))$.

Proof. First Step. First, we discuss the relation between $\beta_{k}(\operatorname{Gin}(I))$ and $\beta_{k}(\operatorname{Lex}(I))$. The same techniques as in Second Step of the proof of Theorem 2.1 yields that

$$
\begin{aligned}
& \operatorname{Gin}(I)=x_{1}\left(H+\left(x_{j}^{2}\right)\right)+x_{1}\left(x_{1}, \ldots, x_{n}\right)^{3} \\
&+x_{2}^{2}\left(G+\left(x_{i} x_{i+1}\right)\right)+\left(x_{1}, \ldots, x_{n}\right)^{5} ; \\
& \operatorname{Lex}(I)=x_{1}\left(H+\left(x_{j-1} x_{j+1}\right)\right)+x_{1}\left(x_{1}, \ldots, x_{n}\right)^{3} \\
&+x_{2}^{2}\left(G+\left(x_{i} x_{i+1}\right)\right)+\left(x_{1}, \ldots, x_{n}\right)^{5} .
\end{aligned}
$$

Since $\operatorname{Gin}(I)_{d}=\operatorname{Lex}(I)_{d}$ for all $d \geq 4$, one has $m_{\leq k}(\operatorname{Gin}(I), d)=$ $m_{\leq k}(\operatorname{Lex}(I), d)$ for all $k$ and for all $d \geq 4$. In addition, $m_{\leq k}(\operatorname{Gin}(I), 3)=$ $m_{\leq k}(\operatorname{Lex}(I), 3)$ for all $k \neq j$ and $m_{\leq j}(\operatorname{Gin}(I), 3)<m_{\leq j}(\operatorname{Lex}(I), 3)$. It then follows from the formula (1) that

$$
\begin{aligned}
\beta_{j+1}(\operatorname{Gin}(I)) & =\beta_{j+1}(\operatorname{Lex}(I)) ; \\
\beta_{k}(\operatorname{Gin}(I)) & <\beta_{k}(\operatorname{Lex}(I))
\end{aligned} \text { for all } \quad k \leq j .
$$


Second Step. Let $\tilde{I}=x_{1} H$. We claim that

$$
\begin{aligned}
\beta_{k, k+3}(I) & =\beta_{k, k+3}(\tilde{I})+\left(\begin{array}{c}
j-2 \\
k
\end{array}\right) ; \\
\beta_{k, k+3}(\operatorname{Gin}(I)) & =\beta_{k, k+3}(\tilde{I})+\left(\begin{array}{c}
j-1 \\
k
\end{array}\right) .
\end{aligned}
$$

Let $I_{\leq d}$ denote the ideal generated by those monomials $u \in I$ with $\operatorname{deg} u \leq$ $d$. Recall that $\beta_{i, i+d}(I)=\beta_{i, i+d}\left(I_{\leq d}\right)\left(\left[10\right.\right.$, Lemma 1.2]). Thus $\beta_{k, k+3}(I)=$ $\beta_{k, k+3}\left(I_{\leq 3}\right)$ and $\beta_{k, k+3}(\operatorname{Gin}(I))=\beta_{k, k+3}\left(\operatorname{Gin}(I)_{\leq 3}\right)$. Since $I_{\leq 3}=x_{1}(H+$ $\left.\left(x_{n}^{2}\right)\right)$ and $\operatorname{Gin}(I)_{\leq 3}=x_{1}\left(H+\left(x_{j}^{2}\right)\right)$, one has $\beta_{k, k+3}\left(I_{\leq 3}\right)=\beta_{k, k+2}((H+$ $\left.\left(x_{n}^{2}\right)\right)$ and $\beta_{k, k+3}\left(\operatorname{Gin}(I)_{\leq 3}\right)=\beta_{k, k+2}\left(\left(H+\left(x_{j}^{2}\right)\right)\right.$. It follows from the same computation as in Third Step of the proof of Theorem 2.1 that

$$
\begin{aligned}
\beta_{k, k+3}(I)=\beta_{k, k+2}\left(\left(H+\left(x_{n}^{2}\right)\right)\right. & =\beta_{k, k+2}(H)+\left(\begin{array}{c}
j-2 \\
k
\end{array}\right) \\
& =\beta_{k, k+3}(\tilde{I})+\left(\begin{array}{c}
j-2 \\
k
\end{array}\right) ; \\
\beta_{k, k+3}(\operatorname{Gin}(I))=\beta_{k, k+2}\left(\left(H+\left(x_{j}^{2}\right)\right)\right. & =\beta_{k, k+2}(H)+\left(\begin{array}{c}
j-1 \\
k
\end{array}\right) \\
& =\beta_{k, k+3}(\tilde{I})+\left(\begin{array}{c}
j-1 \\
k
\end{array}\right) .
\end{aligned}
$$

Third Step. We now turn to the computation of $\beta_{k, k+4}(I)$ and $\beta_{k, k+4}(\operatorname{Gin}(I))$. Let

$$
\begin{aligned}
& J=x_{1}\left(H+\left(x_{j}^{2}\right)\right)+x_{1}\left(x_{1}, \ldots, x_{n}\right)^{3}+x_{2}^{2} G ; \\
& \tilde{J}=x_{1}\left(H+\left(x_{n}^{2}\right)\right)+x_{1}\left(x_{1}, \ldots, x_{n}\right)^{3}+x_{2}^{2} G .
\end{aligned}
$$

We claim that

$$
\beta_{k, k+4}(I)=\beta_{k, k+4}(J)+\left(\begin{array}{c}
i-1 \\
k
\end{array}\right)-\left(\begin{array}{l}
j-1 \\
k+1
\end{array}\right)+\left(\begin{array}{l}
j-2 \\
k+1
\end{array}\right)
$$

$$
\beta_{k, k+4}(\operatorname{Gin}(I))=\beta_{k, k+4}(J)+\left(\begin{array}{l}
i \\
k
\end{array}\right) .
$$

3.1. Since $J_{\leq 3}=\operatorname{Gin}(I)_{\leq 3}$ and $\tilde{J}_{\leq 3}=I_{\leq 3}$, one has $\beta_{k, k+3}(J)=$ $\beta_{k, k+3}(\operatorname{Gin}(I))$ and $\beta_{k, k+3}(\tilde{J})=\beta_{k, k+3}(I)$. Let $J_{\geq d}$ denote the ideal generated by those monomials $u \in J$ with $\operatorname{deg} u \geq d$. Since $J_{\geq 4}$ and $\tilde{J}_{\geq 4}$ are strongly 
stable, the regularity of each of $J$ and $\tilde{J}$ is 4 . Thus, $\operatorname{since} \operatorname{Gin}(\tilde{J})=J$, the cancellation principle $[8$, Corollary 1.21$]$ yields that

$$
\beta_{k, k+4}(J)-\beta_{k, k+4}(\tilde{J})=\beta_{k+1, k+1+3}(J)-\beta_{k+1, k+1+3}(\tilde{J}) .
$$

By virtue of Second Step the right-hand side of (5) is equal to $\left(\begin{array}{l}j-1 \\ k+1\end{array}\right)-\left(\begin{array}{c}j-2 \\ k+1\end{array}\right)$. Thus

$$
\beta_{k, k+4}(J)=\beta_{k, k+4}(\tilde{J})+\left(\begin{array}{l}
j-1 \\
k+1
\end{array}\right)-\left(\begin{array}{l}
j-2 \\
k+1
\end{array}\right) .
$$

3.2. We now show the equality

$$
\beta_{k, k+4}(I)=\beta_{k, k+4}(\tilde{J})+\left(\begin{array}{c}
i-1 \\
k
\end{array}\right) .
$$

Since $I_{\leq 4}=\tilde{J}+\left(x_{2}^{2} x_{n}^{2}\right)$, the short exact sequence

$$
0 \longrightarrow \tilde{J} \longrightarrow I_{\leq 4}=\tilde{J}+\left(x_{2}^{2} x_{n}^{2}\right) \longrightarrow\left(\tilde{J}+\left(x_{2}^{2} x_{n}^{2}\right)\right) / \tilde{J} \longrightarrow 0
$$

yields the long exact sequence

$$
\begin{aligned}
& \longrightarrow\left[\operatorname{Tor}_{k+1}^{A}\left(K,\left(\tilde{J}+\left(x_{2}^{2} x_{n}^{2}\right)\right) / \tilde{J}\right)\right]_{k+1+3} \\
& \longrightarrow\left[\operatorname{Tor}_{k}^{A}(K, \tilde{J})\right]_{k+4} \longrightarrow\left[\operatorname{Tor}_{k}^{A}\left(K, I_{\leq 4}\right)\right]_{k+4} \\
& \longrightarrow\left[\operatorname{Tor}_{k}^{A}\left(K,\left(\tilde{J}+\left(x_{2}^{2} x_{n}^{2}\right)\right) / \tilde{J}\right)\right]_{k+4} \longrightarrow\left[\operatorname{Tor}_{k-1}^{A}(K, \tilde{J})\right]_{k-1+5} \longrightarrow \cdots
\end{aligned}
$$

Since $\left(\left(\tilde{J}+\left(x_{2}^{2} x_{n}^{2}\right)\right) / \tilde{J}\right)_{3}=0$, one has $\left[\operatorname{Tor}_{k+1}^{A}\left(K,\left(\tilde{J}+\left(x_{2}^{2} x_{n}^{2}\right)\right) / \tilde{J}\right)\right]_{k+1+3}=0$. Since the regularity of $\tilde{J}$ is 4 , one has $\left[\operatorname{Tor}_{k-1}^{A}(K, \tilde{J})\right]_{k-1+5}=0$. Thus the above long exact sequence turns out to be

$$
\begin{aligned}
0 \longrightarrow\left[\operatorname{Tor}_{k}^{A}(K, \tilde{J})\right]_{k+4} \longrightarrow & {\left[\operatorname{Tor}_{k}^{A}\left(K, I_{\leq 4}\right)\right]_{k+4} } \\
& \longrightarrow\left[\operatorname{Tor}_{k}^{A}\left(K,\left(\tilde{J}+\left(x_{2}^{2} x_{n}^{2}\right)\right) / \tilde{J}\right)\right]_{k+4} \longrightarrow 0 .
\end{aligned}
$$

In particular

$$
\left.\beta_{k, k+4}\left(I_{\leq 4}\right)\right)=\beta_{k, k+4}(\tilde{J})+\beta_{k, k+4}\left(\left(\tilde{J}+\left(x_{2}^{2} x_{n}^{2}\right)\right) / \tilde{J}\right) .
$$

Since $\beta_{k, k+4}(I)=\beta_{k, k+4}\left(I_{\leq 4}\right)$, to show the equality (7), what we must prove is

$$
\beta_{k, k+4}\left(\left(\tilde{J}+\left(x_{2}^{2} x_{n}^{2}\right)\right) / \tilde{J}\right)=\left(\begin{array}{c}
i-1 \\
k
\end{array}\right) .
$$


Let $\tilde{G}$ denote the ideal generated by those quadratic monomials $x_{p} x_{q}, 1 \leq p<$ $q \leq n$, with $x_{i}^{2} \leq_{\operatorname{lex}} x_{p} x_{q}$. A routine computation shows that

$$
\left(\tilde{J}+\left(x_{2}^{2} x_{n}^{2}\right)\right) / \tilde{J} \cong x_{2}^{2}\left(\left(\tilde{G}+\left(x_{n}^{2}\right)\right) / \tilde{G}\right) .
$$

Thus in particular

$$
\beta_{k, k+4}\left(\left(\tilde{J}+\left(x_{2}^{2} x_{n}^{2}\right)\right) / \tilde{J}\right)=\beta_{k, k+2}\left(\left(\tilde{G}+\left(x_{n}^{2}\right)\right) / \tilde{G}\right) .
$$

Again, the short exact sequence

$$
0 \longrightarrow \tilde{G} \longrightarrow \tilde{G}+\left(x_{n}^{2}\right) \longrightarrow\left(\tilde{G}+\left(x_{n}^{2}\right)\right) / \tilde{G} \longrightarrow 0
$$

yields the long exact sequence

$$
\begin{gathered}
\cdots \longrightarrow\left[\operatorname{Tor}_{k+1}^{A}\left(K,\left(\tilde{G}+\left(x_{n}^{2}\right)\right) / \tilde{G}\right)\right]_{k+1+1} \\
\longrightarrow\left[\operatorname{Tor}_{k}^{A}(K, \tilde{G})\right]_{k+2} \longrightarrow\left[\operatorname{Tor}_{k}^{A}\left(K, \tilde{G}+\left(x_{n}^{2}\right)\right)\right]_{k+2} \\
\longrightarrow\left[\operatorname{Tor}_{k}^{A}\left(K,\left(\tilde{G}+\left(x_{n}^{2}\right)\right) / \tilde{G}\right)\right]_{k+2} \longrightarrow\left[\operatorname{Tor}_{k-1}^{A}(K, \tilde{G})\right]_{k-1+3} \longrightarrow \cdots
\end{gathered}
$$

Since $\left.\left(\tilde{G}+\left(x_{n}^{2}\right)\right) / \tilde{G}\right)_{1}=0$, one has $\left[\operatorname{Tor}_{k+1}^{A}\left(K,\left(\tilde{G}+\left(x_{n}^{2}\right)\right) / \tilde{G}\right)\right]_{k+1+1}=0$. Since the regularity of $\tilde{G}$ is 2 , one has $\left[\operatorname{Tor}_{k-1}^{A}(K, \tilde{G})\right]_{k-1+3}=0$. Thus

$$
\beta_{k, k+2}\left(\left(\tilde{G}+\left(x_{n}^{2}\right)\right) / \tilde{G}\right)=\beta_{k, k+2}\left(\tilde{G}+\left(x_{n}^{2}\right)\right)-\beta_{k, k+2}(\tilde{G}) .
$$

Again, the same computation as in Third Step of the proof of Theorem 2.1 says that

$$
\beta_{k, k+2}\left(\tilde{G}+\left(x_{n}^{2}\right)\right)-\beta_{k, k+2}(\tilde{G})=\left(\begin{array}{c}
i-1 \\
k
\end{array}\right) .
$$

The equalities (9) and (10) together with (11) now yield the desired equality (8).

3.3. The first equality (3) in our claim follows from the equalities (6) and (7). On the other hand, since Gin $(I)$ and $J$ are strongly stable, the second equality (4) in our claim follows from the formula [9, Corollary 3.4 (a)] obtained by Eliahou and Kervaire [7].

Fourth Step. By virtue of Second Step and Third Step, it follows that

$$
\begin{array}{ll}
\beta_{k, k+3}(I)=\beta_{k, k+3}(\operatorname{Gin}(I)) & \text { for all } \quad k \geq j ; \\
\beta_{k, k+4}(I)=\beta_{k, k+4}(\operatorname{Gin}(I)) & \text { for all } \quad k \geq i+1 .
\end{array}
$$

The cancellation principle $[8$, Corollary 1.21$]$ then guarantees that

$$
\beta_{k, k+5}(I)=\beta_{k, k+5}(\operatorname{Gin}(I)) \quad \text { for all } \quad k \geq i .
$$


Since the regularity of each of $I$ and $\operatorname{Gin}(I)$ is 5 , one has $\beta_{k}(I)=\beta_{k}(\operatorname{Gin}(I))$ for all $k \geq i+1$. Again, by virtue of Third Step, it follows that

$$
\beta_{i, i+4}(I)=\beta_{i, i+4}(J)<\beta_{i, i+4}(J)+1=\beta_{i, i+4}(\operatorname{Gin}(I)) .
$$

In particular $\beta_{i}(I)<\beta_{i}(\operatorname{Gin}(I))$. Hence $\beta_{k}(I)<\beta_{k}(\operatorname{Gin}(I))$ for all $k \leq i$.

\section{REFERENCES}

1. Aramova, A., Herzog, J., and Hibi, T., Ideals with stable Betti numbers, Adv. Math. 152 (2000), 72-77.

2. Bigatti, A., Upper bounds for Betti numbers of a given Hilbert function, Comm. Alg. 21 (1993), 2317-2334.

3. Bruns, W., and Herzog, J., Cohen-Macaulay rings, Revised Edition, Cambridge University Press, 1996.

4. Conca, A., Reduction numbers and initial ideals, Proc. Amer. Math. Soc. 131 (2003), 10151020.

5. Conca, A., Herzog, J., and Hibi, T., Rigid resolutions and big Betti numbers, Comment. Math. Helv. 79 (2004), 826-839.

6. Eisenbud, D., Commutative Algebra with a View toward Algebraic Geometry, Springer-Verlag, 1995.

7. Eliahou, S., and Kervaire, M., Minimal resolutions of some monomial ideals, J. Algebra 129 (1990), 1-25.

8. Green, M., Generic initial ideals, in Six Lectures on Commutative Algebra (J. Elias, J. M. Giral, R. M. Miro-Roig and S. Zarzuela, Eds.), Birkhäuser, 1998, 119-186.

9. Herzog, J., Generic initial ideals and graded Betti numbers, in Computational Commutative Algebra and Combinatorics (T. Hibi, Ed.), Adv. Stud. Pure Math. 33 (2002), 75-120.

10. Herzog, J., and Hibi, T., Componentwise linear ideals, Nagoya Math. J. 153 (1999), 141-153.

11. Hulett, H., Maximal Betti numbers of homogeneous ideals with a given Hilbert function, Comm. Alg. 21 (1993), 2335-2350.

DEPARTMENT OF PURE AND APPLIED MATHEMATICS

GRADUATE SCHOOL OF INFORMATION SCIENCE AND TECHNOLOGY

OSAKA UNIVERSITY

TOYONAKA, OSAKA 560-0043

JAPAN

E-mail: s-murai@ist.osaka-u.ac.jp, hibi@math.sci.osaka-u.ac.jp 